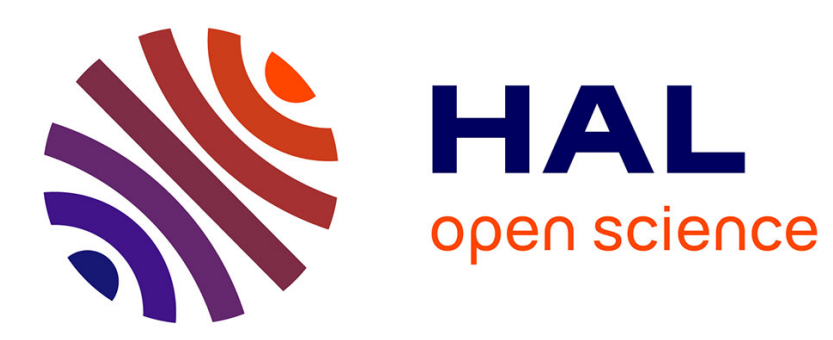

\title{
Ab initio prediction of a negative Barkas coefficient for slow protons and antiprotons in $\mathrm{LiF}$
}

Xixi Qi, Fabien Bruneval, Ivan Maliyov

\section{To cite this version:}

Xixi Qi, Fabien Bruneval, Ivan Maliyov. Ab initio prediction of a negative Barkas coefficient for slow protons and antiprotons in LiF. Physical Review Letters, 2022, 128 (4), pp.043401. 10.1103/PhysRevLett.128.043401 . cea-03552249

\section{HAL Id: cea-03552249 https://hal-cea.archives-ouvertes.fr/cea-03552249}

Submitted on 2 Feb 2022

HAL is a multi-disciplinary open access archive for the deposit and dissemination of scientific research documents, whether they are published or not. The documents may come from teaching and research institutions in France or abroad, or from public or private research centers.
L'archive ouverte pluridisciplinaire HAL, est destinée au dépôt et à la diffusion de documents scientifiques de niveau recherche, publiés ou non, émanant des établissements d'enseignement et de recherche français ou étrangers, des laboratoires publics ou privés. 


\title{
$A b$ Initio Prediction of a Negative Barkas Coefficient for Slow Protons and Antiprotons in LiF
}

\author{
Xixi Qi॰ and Fabien Bruneval『 \\ Université Paris-Saclay, CEA, Service de Recherches de Métallurgie Physique, 91191 Gif-sur-Yvette, France \\ Ivan Maliyov \\ Department of Applied Physics and Materials Science, California Institute of Technology, Pasadena, California 91125, USA
}

(Received 23 September 2021; accepted 5 January 2022; published 24 January 2022)

\begin{abstract}
We report the $a b$ initio prediction of a negative Barkas coefficient in lithium fluoride (LiF) insulator at low velocity $\left(v<0.25\right.$ a.u., $\left.E_{\mathrm{kin}} \sim 2 \mathrm{keV}\right)$. The electronic stopping power of protons in $\mathrm{LiF}$ has been extensively studied both experimentally and theoretically because of a controversial threshold effect. While our time-dependent density-functional theory simulations confirm the presence of a velocity threshold below which the proton stopping power vanishes, our calculations demonstrate that the antiprotons do not experience such a threshold. The combination of those two contrasting behaviors gives rise to an unprecedented negative Barkas effect: the stopping power of antiprotons is larger than that of protons. We identify that the slow antiproton at close encounter destabilizes a $p$ orbital of the $\mathrm{F}^{-}$anion pointing toward the antiproton. This particular orbital becomes highly polarizable and hence contributes much to the stopping power.
\end{abstract}

DOI: 10.1103/PhysRevLett.128.043401

The interaction between swift charged particles and matter has been a central topic since the earliest days of quantum mechanics. Famous researchers, such as Bohr [1], Bethe [2], or Lindhard [3], have developed models to quantify the energy transfer between the charged projectile and the target atoms. While tightly bound to the development of particle physics, this interaction is still key to modern applications: nuclear materials [4], radiotherapy [5], space-grade materials [6].

The interaction between a charged particle and a material is best characterized by the so-called stopping power $S$ [7]:

$$
S=-\frac{d E_{\mathrm{kin}}}{d z}
$$

which measures the average decrease in kinetic energy of the projectile $E_{\text {kin }}$ as a function of the path length $z$. Most of the stopping power occurs through the electronic excitations in the target material, named the electronic stopping power $S_{e}$. But the classical nuclear-nuclear collision term $S_{n}$ may also produce a finite contribution at low velocity.

In many cases, the electronic stopping power (ESP) of materials can be qualitatively understood using the historical model of Lindhard [8,9], which considers a noninteracting homogeneous electron gas within the linearresponse (LR) approximation. However, some subtle effects do not obey this simple treatment. Let us highlight two such features.

First, Barkas and co-workers [10] measured in the 1950s that the stopping power of negative pions was noticeably lower than that of positive pions: this is known as the "Barkas effect." This difference contradicts the LR approximation that predicts a quadratic dependence with respect to the projectile charge $Z_{1}$ and therefore that makes no difference between a proton and its antiparticle. The next coefficient in a polynomial expansion with respect to $Z_{1}$, the cubic term, is named the Barkas coefficient and is always measured being positive in practice [11,12].

Second, in the low-projectile-velocity regime, the Lindhard model predicts $S_{e} \propto v$, a behavior indeed observed in metallic targets. However, for insulators, there has been a passionate debate over the years about the existence of a threshold velocity below which the electronic stopping power vanishes. Lithium fluoride, with its wide band gap of $13.6 \mathrm{eV}$ [13], was the battleground for this controversy to which both theoreticians and experimentalists participated: some advocating for the absence of threshold [14,15], and others for the presence of a threshold [16-18].

Today, the existence of a threshold is being supported by the latest experiments at very low velocity [18], but also by a new numerical approach in this field, the $a b$ initio calculations within time-dependent density-functional theory (TDDFT) [19]. The seminal work of Pruneda et al. 20]] using real-time TDDFT (RT TDDFT), proved the existence of a threshold for proton in LiF. Following this original work that demonstrated the predictive power of $a b$ initio calculations, a wealth of new TDDFT studies on other materials bloomed [21-29].

In this Letter, we employ state-of-the-art RT TDDFT to simulate proton and antiproton irradiation of LiF. While our 
simulations reproduce the available experimental data, we also explore the low-velocity regime where experimental results are scarce. We confirm the presence of a velocity threshold in the ESP of protons, but we predict that no such threshold exists for antiprotons. These two opposite behaviors add up to induce a negative Barkas coefficient for velocities below 0.25 a.u.: the ESP of antiproton is actually larger than that of proton. We then analyze the origin of this unique behavior thanks to the electronic structure of the combined system of $\mathrm{LiF}$ and of the charged projectile.

Method.-In our study, the ESP is evaluated from RT TDDFT simulations, in which a charged projectile (proton $\mathrm{H}^{+}$or antiproton $\overline{\mathrm{H}}^{-}$) follows a linear trajectory at constant velocity $v$ through a cluster of immobile $\mathrm{LiF}$ atoms. This particular setup is customary to ESP calculations [20,30,31] and to atomic collisions [32]. Let us detail here the procedure.

Performing calculations using a straight-line trajectory with frozen-atom targets allows us to disentangle the electronic part of the stopping power $S_{e}$ from the nuclear part $S_{n}$. The straight-line trajectory is also computationally advantageous since it permits us to give the cluster the correct cylindrical shape that will embed the projectile during its entire course. The nuclear stopping power $S_{n}$ will be added up later using classical mechanics [33].

Figure 1 shows a typical example of our simulations. The upper panel displays the actual cylindrical cluster we employ. It consists of 126 atoms cut from the LiF rocksalt structure with the experimental lattice constant. The velocity and the cylinder axis are along the $\langle 111\rangle$ direction. The cylinder length is approximately $22 \AA$ and its diameter is around $6 \AA$. The axis of the cylinder is centered on an atomic column, so that the impact parameter $p=0$ corresponds to a knock-on collision. This allows us to use the polar symmetry as described in Ref. [26]. Four different impact parameters are considered: $p=0.225$, $0.45,0.675$, and $0.90 \AA$, so that we could average them with the geometric weight induced by the polar symmetry to obtain the so-called random electronic stopping power (RESP). The reliability of this sampling is demonstrated in Supplemental Material [34] where using eight impact parameters leads to the same results. In Supplemental Material, we also report RESP calculations for the $\langle 001\rangle$ direction, with very similar results. This confirms the directional insensitivity of RESP. Finally, the cluster size convergence is proven in Supplemental Material.

It is worth noting that at low projectile velocities $(v<0.2$ a.u. $)$ and impact parameters $(p<0.1 \AA)$, the actual projectile trajectories would deviate from the linear ones: protons are repelled and antiprotons are attracted by target nuclei. However, this deviation is weak (as shown in Supplemental Material) and would just affect the few trajectories that come very close to the nuclei. Trajectories with $p<0.1 \AA$ only account for $1.5 \%$ of the

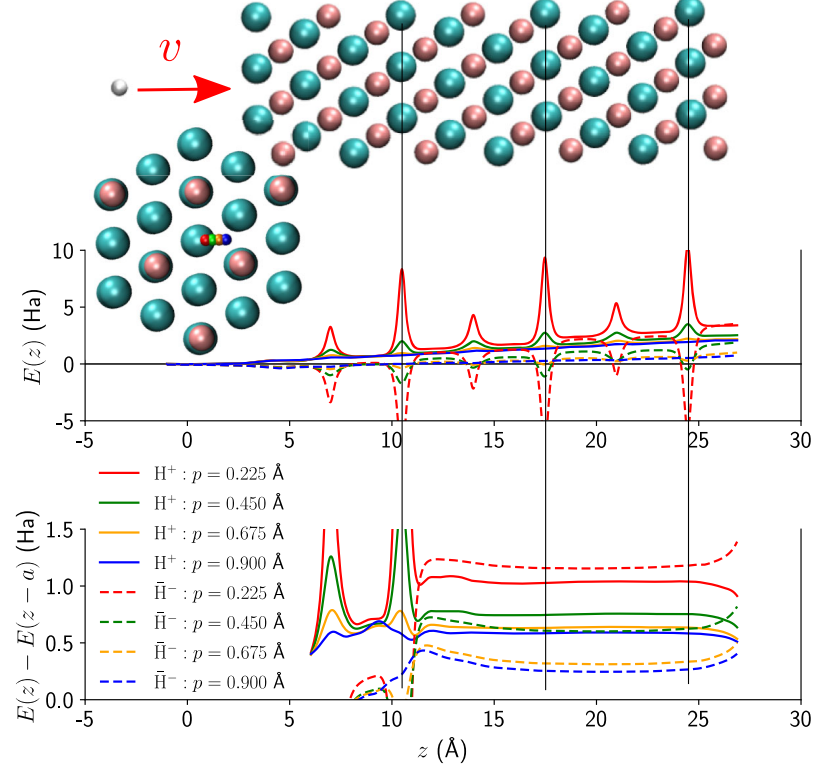

FIG. 1. Theoretical model for the LiF crystal. Upper panel: LiF cluster of 126 atoms with longitudinal and transversal views, extracted from the perfect rocksalt structure in a cylindrical shape along the $\langle 111\rangle$ axis. Cyan, pink, and white balls represent, respectively, $\mathrm{Li}, \mathrm{F}$, and the projectile. In the transversal view, the four impact parameters used in this study are shown in red, green, orange, and blue. Middle panel: Evolution of the total energy of the electrons $E(z)$ as a function of the penetration depth $z$. The zero was set to the value at $t=0$. Lower panel: Total electronic energy difference $E(z)-E(z-a)$, where $a$ is the periodicity length. In the middle and lower panels, the total energies are exhibited for $v=0.4$ a.u. for four impact parameters $p$ and two projectiles: proton (solid lines) and antiproton (dashed lines).

total trajectories in the impact parameter average we perform to obtain the RESP.

Note that antiprotons at small velocities and very small impact parameters have a finite probability of annihilating with one of the protons of target nuclei. This is not taken into account in our simulations, but again the geometrical weight of those trajectories is small and has little influence on the final impact-parameter-averaged value.

In the central panel of Fig. 1, we report the total energy of the electrons $E(z)$ for the four impact parameters, for protons and antiprotons. $E(z)$ increases along with the penetration depth $z$, with marked peaks when the projectile passes nearby the target nuclei. As the motion of the atoms in the simulation does not obey the Newton law of mechanics, the total energy is, of course, not conserved. However, one can show that the total electronic energy increase per unit length is precisely the negative of the ESP [30]:

$$
S_{e}=-\lim _{z \rightarrow+\infty} \frac{E(z)-E(z-a)}{a},
$$

where $a$ is the lattice periodicity. Here, in direction $\langle 111\rangle$, $a$ is $6.989 \AA$. When a stationary regime is achieved, the $z$ 
dependence of $E(z)-E(z-a)$ vanishes and the limit to large $z$ is achieved in Eq. (2). In the lower panel of Fig. 1, we plot the energy increase on a lattice period, as needed for Eq. (2). After one period, this energy difference stabilizes to give a constant estimate of the energy increase as can be seen around $z=20 \AA$ in the lower panel of Fig. 1. Note that when the projectile approaches the exit of the cluster, the energy difference starts to vary again.

In practice, we employ the localized Gaussian-type orbital code MOLGW [38] within the adiabatic local-density approximation (ALDA). We use very complete basis sets, namely aug-cc-pCVQZ, for the atoms that are close to the projectile track. We had shown previously [28] that this is necessary for a quantitative description of ESP. Those basis sets contain basis functions with high angular momentum (up to $l=4$ ), diffuse functions, and also valence and core polarization functions. The rest of the atoms can be described with a lighter basis (cc-pVDZ). Here, 48 atoms have the most complete basis and 78 atoms have the simpler one. The projectile itself is a simple point charge with no basis function. The time-dependent Kohn-Sham equations are integrated with the Magnus 2 propagator, combined with a predictor-corrector procedure [39]. The time step comprises between 0.05 and 0.10 a.u. depending on the projectile velocity. In addition, we have performed a few calculations using a tuned hybrid functional (PBEh) observing a very small effect. This confirms the weak sensitivity to the exchange-correlation approximation already highlighted in Refs. [40] and [26]. These hybrid functional calculations are reported in Supplemental Material. Finally, ALDA neglects the memory effects in the exchange correlation. This approximation has been shown to hold for weakly charged ions in the homogeneous electron gas [41].

Negative Barkas coefficient.-In Fig. 2, we report the RT TDDFT calculation of RESP in LiF for impinging protons and antiprotons, together with the available experimental points. For comparison, we also report a linear-response TDDFT (LR TDDFT) curve obtained with ABINIT $[42,43]$.

First of all, the overall agreement between RT TDDFT and experimental data is good for proton and antiproton. The positive Barkas effect is obtained for velocities above $v=0.3$ a.u., as expected [7]. LR TDDFT calculation predicts a threshold, however, for both the proton and the antiproton, because of its simple $Z_{1}^{2}$ charge dependence. For proton, RT TDDFT yields a major improvement compared to LR TDDFT: the presence of a velocity threshold around $v=0.1$ a.u. confirms the newest experimental data [18] and the previous RT TDDFT study of Pruneda and co-workers [20].

Now focusing on antiprotons, our RT TDDFT calculations show no velocity threshold. The RESP shows a complex line shape at low velocities: a plateau in between 0.15 and 0.30 a.u. and a linear behavior $S_{e} \propto v$ below 0.15 a.u. As no experimental points are available in this range, our calculations can be considered as ab initio predictions.

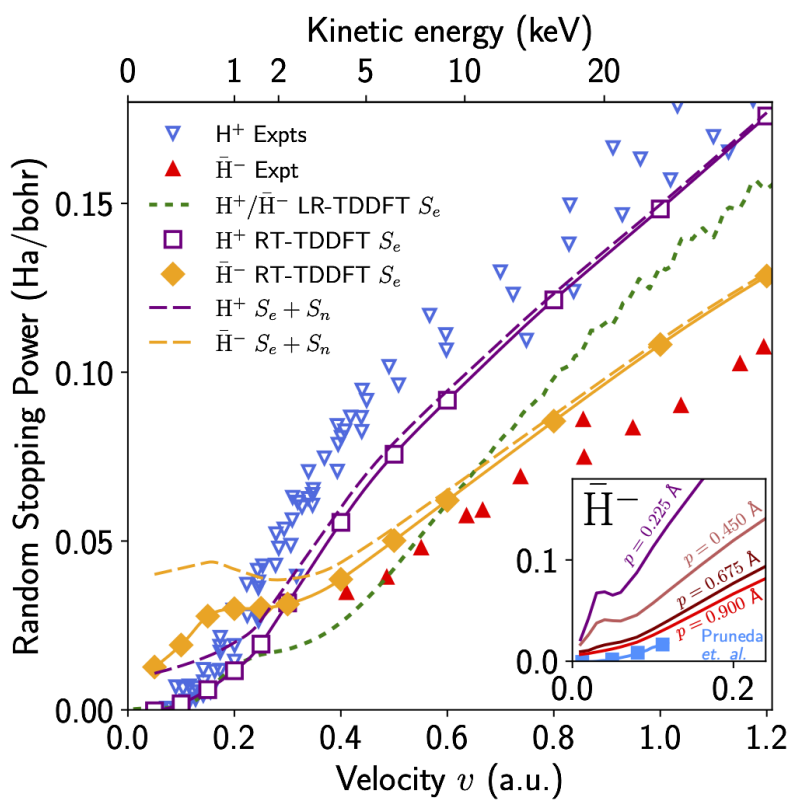

FIG. 2. LiF random stopping power for protons and antiprotons in the low-velocity regime (below $30 \mathrm{keV}$ ). LR TDDFT that is insensitive to the projectile charge sign is represented with a short-dashed line. The solid lines with symbols are RT TDDFT $S_{e}$. The calculated total random stopping power, including both the electronic and the nuclear parts $S_{e}+S_{n}$, is drawn with the long-dashed lines. Several experiments are reported jointly $[15,17,18]$ (triangles). The inset represents the RT TDDFTcalculated ESP for antiprotons for each of the four impact parameters, together with the earlier work of Pruneda et al. [20].

Our results contrast much with the conclusions of the earlier RT TDDFT calculations of Pruneda and co-workers [20]. In the inset of Fig. 2, we report the ESP for each impact parameter $p$ individually, together with the results of Ref. [20], that selected a single impact parameter under the $\langle 110\rangle$ channeling condition. Our results for the large impact parameter $p=0.90 \AA$ confirm their calculations: the antiproton stopping is very low in the channeling condition. However, the complex behavior arises for the closer impacts at $p=0.225$ and $0.45 \AA$. We conclude then that the RESP of antiproton, which is a weighted average of all possible impacts, is indeed dominated by the close and intermediate impacts at low velocity.

Thanks to this strong proton-antiproton asymmetry, we witness the occurrence of the elusive negative Barkas coefficient: the ESP of proton is lower than that of antiproton for $v$ below 0.25 a.u. The possibility of such a negative coefficient had been foreseen in models, such as in a harmonic oscillator [44], but never observed, to the best of our knowledge, in any experiment or in any $a b$ initio calculation.

So far we concentrated on the electronic contribution. Recently, Nordlund and co-workers [45] showed that there also exists a proton-antiproton asymmetry for the nuclear stopping power $S_{n}$. We therefore implemented their 
approach to obtain the antiproton nuclear stopping power in LiF, $S_{n}$, in the binary collision approximation [33]. Details are reported in Supplemental Material. The proton contribution comes from Ref. [33]. Finally, summing up the nuclear and electronic contributions produces an even more negative Barkas coefficient, as shown in Fig. 2.

Fractional charges analysis. - We can further characterize the charge asymmetry in LiF by performing calculations for fractional projectile charges. In Fig. 3, we report the RESP of LiF as a function of $Z_{1}$ for three velocities. LR TDDFT has a pure $\left(Z_{1}\right)^{2}$ behavior. At the highest velocity, $v=1.2$ a.u., the RT TDDFT calculations follow the same parabolic shape, which confirms the LR approximation for this velocity. Just above the velocity threshold, at $v=0.4$ a.u., the RT TDDFT starts to depart from the LR but still with a positive Barkas coefficient. However, around the threshold, at $v=0.2$ a.u., the LR or even any low-order polynomial expansion in $Z_{1}$ are not able to capture the complex $Z_{1}$ dependence. For low positive values of $Z_{1}$, the RESP vanishes. For negative $Z_{1}$, RT TDDFT is almost three times larger than the LR TDDFT and a negative Barkas coefficient is realized. At low velocity, the classical low-order polynomial expansion should be abandoned. We nevertheless keep the expression "Barkas coefficient" in this study for consistency with the historical naming.

Isolated ions analysis. - We would like to understand which particular feature in the electronic structure of $\mathrm{LiF}$ is key to the negative Barkas coefficient. To simplify the problem, we have performed RT TDDFT trajectories on lithium cations and fluorine anions and found that at low velocities, $\mathrm{F}^{-}$was responsible for most of the stopping power. More precisely, by projecting the time-dependent Kohn-Sham orbitals on the self-consistent orbitals, we

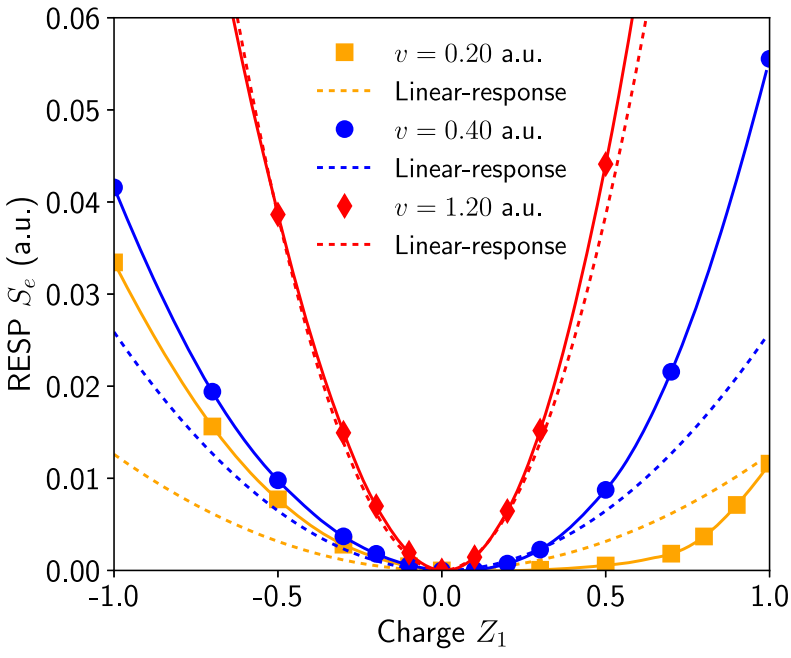

FIG. 3. LiF RESP calculated when continuously varying the projectile charge $Z_{1}$ from -1 (antiproton) to 1 (proton). Solid lines and symbols are the RT TDDFT calculations, whereas the dashed lines are the LR TDDFT results. observe the depletion of the $p_{x}$ and $p_{z}$ orbitals of $\mathrm{F}^{-}$. Here, $z$ stands for the direction of the velocity and $x$ for the impact parameter direction. This figure can be found in Supplemental Material [34].

RT TDDFT are our most accurate results in this study, however, they cannot be easily interpreted. We now turn to LR TDDFT, not for quantitative results, but just to gain insights. In the low-velocity limit, let us assume that the electronic system is allowed enough time to adapt to the perturbing projectile. Then we consider an adiabatic model in which a proton or an antiproton is fixed at a small distance $\left(p=0.45 \AA\right.$ ) of an $\mathrm{F}^{-}$anion $[14,46]$. The precise setup is represented in the inset of Fig. 4, where positive charges are also added so to mimic the $6 \mathrm{Li}^{+}$first nearest neighbors.

Figure 4 shows the first excitation energy as obtained from LR TDDFT within ALDA. We see the increase of the excitation energy in the presence of a positive proton and its strong decrease in the presence of a negative antiproton in agreement with Ref. [46]. Indeed the extra proton is much attractive for the fluorine electrons, which become very stable and then not polarizable at all. The extra antiproton, quite the contrary, repels much of the $\mathrm{F}^{-}$electrons. In particular, the $p$ orbital that points toward the antiproton is much destabilized. This orbital is referred to as $p_{u}$, with $\hat{u}$ the unit vector pointing to the antiproton. It is graphically represented in Fig. 4.

We would like to see if this orbital is indeed the cause of the increased stopping power at low velocity. The oscillator strengths one usually calculates for optical excitations have no link to their contribution to the ESP. Instead, we use the stopping cross-section (SCS) of a finite system [47],

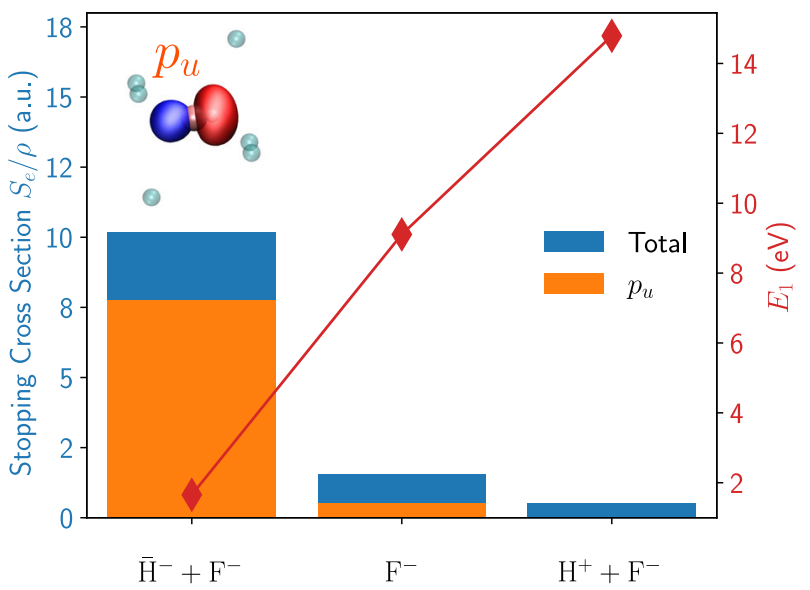

FIG. 4. $\mathrm{F}^{-}$anion stopping cross section (SCS) $S_{e} / \rho$ at $v=$ 0.2 a.u. (bars, left axis) and first excitation energy (diamond, right axis). The fluorine anion is considered in three different situations: alone, with a proton $\mathrm{H}^{+}$at distance $p=0.45 \AA$, or with an antiproton $\overline{\mathrm{H}}^{-}$at the same distance. The $p_{u}$ orbital in the presence of an antiproton in the direction $u$ is drawn in an inset. The stopping cross section that originates from the $p_{u}$ orbital is separated from the rest. 
which is calculated for $v=0.2$ a.u. with the bars in Fig. 4 for the three systems under scrutiny. SCS derives directly from generalized oscillator strengths [47], which are velocity-dependent quantities. SCS measures the intrinsic ability of a system to capture energy from a passing charged projectile. The simple model is in qualitative agreement with the complete rocksalt $\mathrm{LiF}$ calculations reported above: the presence of the proton decreases the stopping power, whereas the antiproton amplifies it by a factor of 10 . With the LR, one can identify each contribution to the stopping power individually: the orange bars in Fig. 4 highlight the stopping power that arises from the excitations of the $p_{u}$ orbital defined above. The $p_{u}$ orbital destabilized by the antiproton is identified as the main contributor to the stopping at low velocity. This statement is in agreement with the complete RT TDDFT calculations where the $p_{x}$ and $p_{z}$ orbitals are depleted: indeed, the unit vector $\hat{u}$ is contained in the $(x z)$ plane of the collision. At larger velocity, all the valence orbitals contribute to the stopping power and the particular role of the $p_{u}$ orbital of $\mathrm{F}^{-}$is lost. These data can be found in Supplemental Material [34].

Conclusion.-In conclusion, this Letter reports a negative Barkas coefficient for protons and antiprotons below $v<0.25$ a.u. $(2 \mathrm{keV})$ in LiF, obtained from RT TDDFT simulations. Whereas the RESP for proton experiences a threshold effect, the one of antiproton does not. The combination of the two contrasting behaviors induces a negative Barkas effect, which had never been observed yet in experiments or in ab initio calculations, to the best of our knowledge.

This unique feature originates from the small impact parameters when the antiproton approaches the $\mathrm{F}^{-}$anions. Using fractional charges, as only possible in simulations, we showed that the $Z_{1}$ behavior of the RESP cannot be accounted for with low-order polynomials in the lowvelocity regime, in contrast with the common practice. At close range and low velocity, the electrons of the $\mathrm{F}^{-}$ target are destabilized by the antiproton and become highly polarizable. This is particularly true for the $p$ orbital that points toward the projectile, which is found responsible for about $80 \%$ of the stopping.

We hope this ab initio prediction will stimulate new experimental investigations of antiprotons in insulators in the low-velocity regime in the future.

This work was performed using HPC resources from GENCI-TGCC (Grant No. 2021-096018). We are indebted to I. Nagy for having pointed us to the LiF electronic stopping power.

[1] N. Bohr, Philos. Mag. 25, 10 (1913).

[2] H. Bethe, Ann. Phys. 397, 325 (1930).

[3] J. Lindhard, Mat. Fys. Medd. K. Dan. Vidensk. Selsk 28, 1 (1954).
[4] L. Beck, Y. Serruys, S. Miro, P. Trocellier, E. Bordas, F. Leprêtre, D. Brimbal, T. Loussouarn, H. Martin, S. Vaubaillon, S. Pellegrino, and D. Bachiller-Perea, J. Mater. Res. 30, 1183 (2015).

[5] W. D. Newhauser and R. Zhang, Phys. Med. Biol. 60, R155 (2015).

[6] N. E. Koval, F. Da Pieve, and E. Artacho, R. Soc. Open Sci. 7, 200925 (2020).

[7] P. Sigmund, Particle Penetration and Radiation EffectsGeneral Aspects and Stopping of Swift Point Charges (Springer-Verlag, Berlin, Heidelberg, 2006).

[8] J. Lindhard and M. Scharff, Mat. Fys. Medd. K. Dan. Vidensk. Selsk 27, 1 (1953).

[9] G. J. Iafrate and J. F. Ziegler, J. Appl. Phys. 50, 5579 (1979).

[10] W. H. Barkas, W. Birnbaum, and F. M. Smith, Phys. Rev. 101, 778 (1956).

[11] P. Sigmund and A. Schinner, Nucl. Instrum. Methods Phys. Res., Sect. B 212, 110 (2003).

[12] I. Nagy, A. Arnau, P. M. Echenique, and E. Zaremba, Phys. Rev. B 40, 11983 (1989).

[13] D. Roessler and W. Walker, J. Phys. Chem. Solids 28, 1507 (1967).

[14] K. Eder, D. Semrad, P. Bauer, R. Golser, P. Maier-Komor, F. Aumayr, M. Peñalba, A. Arnau, J. M. Ugalde, and P. M. Echenique, Phys. Rev. Lett. 79, 4112 (1997).

[15] S. P. Møller, A. Csete, T. Ichioka, H. Knudsen, U. I. Uggerhøj, and H.H. Andersen, Phys. Rev. Lett. 93, 042502 (2004).

[16] C. Auth, A. Mertens, H. Winter, and A. Borisov, Phys. Rev. Lett. 81, 4831 (1998).

[17] M. Draxler, S. P. Chenakin, S. N. Markin, and P. Bauer, Phys. Rev. Lett. 95, 113201 (2005).

[18] S. N. Markin, D. Primetzhofer, and P. Bauer, Phys. Rev. Lett. 103, 113201 (2009).

[19] E. Runge and E. K. U. Gross, Phys. Rev. Lett. 52, 997 (1984).

[20] J. M. Pruneda, D. Sánchez-Portal, A. Arnau, J. I. Juaristi, and E. Artacho, Phys. Rev. Lett. 99, 235501 (2007).

[21] A. A. Correa, J. Kohanoff, E. Artacho, D. Sánchez-Portal, and A. Caro, Phys. Rev. Lett. 108, 213201 (2012).

[22] M. A. Zeb, J. Kohanoff, D. Sánchez-Portal, A. Arnau, J. I. Juaristi, and E. Artacho, Phys. Rev. Lett. 108, 225504 (2012).

[23] A. Schleife, E. W. Draeger, Y. Kanai, and A. A. Correa, J. Chem. Phys. 137, 22A546 (2012).

[24] A. Lim, W. M. C. Foulkes, A. P. Horsfield, D. R. Mason, A. Schleife, E. W. Draeger, and A. A. Correa, Phys. Rev. Lett. 116, 043201 (2016).

[25] J. Kohanoff and E. Artacho, PLoS One 12, e0171820 (2017).

[26] I. Maliyov, J.-P. Crocombette, and F. Bruneval, Eur. Phys. J. B 91, 172 (2018).

[27] Y. Yao, D. C. Yost, and Y. Kanai, Phys. Rev. Lett. 123, 066401 (2019).

[28] I. Maliyov, J.-P. Crocombette, and F. Bruneval, Phys. Rev. B 101, 035136 (2020).

[29] R. Tandiana, C. Clavaguéra, K. Hasnaoui, PedrozaMontero, J. Naín, and A. de la Lande, Theor. Chem. Acc. 140, 126 (2021).

[30] A. A. Correa, Comput. Mater. Sci. 150, 291 (2018). 
[31] C. Shepard, R. Zhou, D. C. Yost, Y. Yao, and Y. Kanai, J. Chem. Phys. 155, 100901 (2021).

[32] N. Sisourat, I. Pilskog, and A. Dubois, Phys. Rev. A 84, 052722 (2011).

[33] J. F. Ziegler, J. P. Biersack, and U. Littmark, The Stopping and Ranges of Ions in Matter (Pergamon, New York, 1985).

[34] See Supplemental Material at http://link.aps.org/ supplemental/10.1103/PhysRevLett.128.043401 for additional numerical and convergence details, which includes Refs. [35-37].

[35] P. Borlido, T. Aull, A. W. Huran, F. Tran, M. A. L. Marques, and S. Botti, J. Chem. Theory Comput. 15, 5069 (2019).

[36] S. Refaely-Abramson, M. Jain, S. Sharifzadeh, J. B. Neaton, and L. Kronik, Phys. Rev. B 92, 081204(R) (2015).

[37] J. F. Ziegler, M. Ziegler, and J. Biersack, Nucl. Instrum. Methods Phys. Res., Sect. B 268, 1818 (2010).

[38] F. Bruneval, T. Rangel, S. M. Hamed, M. Shao, C. Yang, and J. B. Neaton, Comput. Phys. Commun. 208, 149 (2016).
[39] A. Castro, M. A. L. Marques, and A. Rubio, J. Chem. Phys. 121, 3425 (2004).

[40] K. G. Reeves, Y. Yao, and Y. Kanai, Phys. Rev. B 94, 041108(R) (2016).

[41] V. U. Nazarov, J. M. Pitarke, C. S. Kim, and Y. Takada, Phys. Rev. B 71, 121106(R) (2005).

[42] A. A. Shukri, F. Bruneval, and L. Reining, Phys. Rev. B 93, 035128 (2016).

[43] X. Gonze et al., Comput. Phys. Commun. 205, 106 (2016).

[44] H. H. Mikkelsen and P. Sigmund, Phys. Rev. A 40, 101 (1989).

[45] K. Nordlund, D. Sundholm, P. Pyykkö, D. M. Zambrano, and F. Djurabekova, Phys. Rev. A 96, 042717 (2017).

[46] B. Solleder, L. Wirtz, and J. Burgdörfer, Phys. Rev. B 79, 125107 (2009).

[47] E. H. Mortensen, J. Oddershede, and J. R. Sabin, Nucl. Instrum. Methods Phys. Res., Sect. B 69, 24 (1992). 